\title{
Sozialfiguren der Corona-Pandemie
}

blog.kulturwissenschaften.de/sozialfiguren-der-corona-pandemie/

$\underline{\text { Sebastian J. MoserTobias SchlechtriemenSozialfiguren der Corona-Pandemie }}$ Ein Aufschlag

Man kann bestreiten, wie Andreas Reckwitz es kürzlich in einem Interview mit der Berliner Zeitung getan hat, ${ }^{1}$ dass die Corona-Pandemie einen Epochenbruch darstellt. Unzweifelhaft ist hingegen, dass die aktuelle Pandemie und die mit ihr einhergehenden Maßnahmen den Alltag der Menschen weltweit grundlegend verändert haben. Während der Blick auf Brüche - meist aus der zeitlichen Distanz danach - ein klares Vorher und Nachher erkennen lässt, stecken wir derzeit mittendrin. Wir befinden uns in einem Schwebezustand zwischen „nicht mehr“ und „noch nicht“ oder „wieder“. Genau in solchen unsicheren Zwischenzeiten betreten Sozialfiguren die gesellschaftliche Bühne. ${ }^{2}$ Sie verkörpern in figurativer Form, welche gesellschaftlichen Erfahrungs- und Problemlagen im Alltag ,unter den Nägeln“ brennen und ermöglichen uns als Gesellschaft eine Verständigung darüber.

Die Pandemie brennt geradezu ein ganzes Feuerwerk von Sozialfiguren ab vermutlich auch ein Hinweis auf das Ausmaß und den Ernst der Lage. Am Anfang war bekanntlich der mit Bergen von Klopapier den Supermarkt verlassende Hamsterkäufer, bereit zur Einigelung auf unbestimmte Zeit. Im Verlauf der Pandemie lernte die Bevölkerung dann Namen von bisher öffentlich weitestgehend unbekannten Virologen - vor allem männlichen - oder anderen wissenschaftlichen Expert*innen kennen, die zur jeweiligen politischen Couleur oder Weltanschauung derjenigen passten, die sich auf ihre Argumente stützten. Zugleich erhielten Namen wie jener des Bayerischen Unternehmens Webasto, des österreichischen Skiorts Ischgl oder des Huanan-Marktes in der chinesischen Metropole Wuhan traurige Berühmtheit, weil es dort zum Auftritt von Patient*in o kam. Diese Figur war nicht nur krank, sondern ansteckend und provozierte genau deswegen Fragen nach dem richtigen Verhalten, nach Schuld und Verantwortung. Fragen ähnlicher Art, vor allem von Verantwortungslosigkeit und asozialem Verhalten, verdichteten sich im Verlauf der Pandemie in den sogenannten Maskenverweigerern.

In all den genannten Fällen hatten die Figuren eine menschliche Gestalt, einen Namen, ein Alter, ein Geschlecht, was half, ihre Geschichte erzählbar und damit anschaulich zu machen. Allerdings werden sie im Rahmen eines sozialfigurativen Ansatzes nicht als individuelle Persönlichkeiten adressiert. Vielmehr interessiert das Gesellschaftliche, das in ihnen steckt. Dies gilt ebenso für die Söders, Laschets oder Kretschmanns. Hier geht es nicht um konkrete Personen, sondern darum, wie diese die Position des Ministerpräsidenten durch die Figur des paternalistischen und volksnahen Landesvaters zur Aufführung bringen. Neben weiteren Sozialfiguren wie 
der Risikogruppe, den Systemrelevanten oder den Balkonkünstler*innen wird in dieser KWI-Blog-Reihe auch von solchen Figuren zu reden sein, in denen sich eher soziale Praktiken wie das Absagen verdichten, die ebenfalls den pandemischen Alltag prägen.

In den besagten historischen Zwischenzeiten schießen Sozialfiguren - Pilzen gleich aus dem Boden. Dadurch sind sie eng mit der Gegenwart verbunden und entfalten für die Zeitgenoss*innen eine affizierende Wirkung. Sind sie erst einmal in aller Munde, erscheint ihr Auftreten den Gesellschaftsmitgliedern zudem höchst evident. Gleichzeitig hätte man sie jedoch nur schwerlich (oder gar nicht) voraussagen können. Dass Sozialfiguren einen Zeitindex aufweisen, bedeutet auch, dass die von ihnen ausgelösten Emotionen verebben, sobald sie wieder verschwinden oder sich gar institutionalisieren. Außerdem kommt eine Sozialfigur selten allein. Sie bewegt sich zumeist in einem Gefüge mit anderen Begleit- und Nachbarfiguren oder auch Gegenspieler- und Kontrastfiguren, die ihre Konturen maßgeblich mitprägen. Um sich jedoch nicht des Rückzugs auf die Gegenwart schuldig zu machen, den Norbert Elias seinen soziologischen Kolleg*innen einst vorwarf, ${ }^{3}$ muss erwähnt werden, dass sich die figurative Konstellation nicht auf die Gegenwart beschränkt, sondern historische Vorläuferfiguren (Präfigurationen) miteinschließt. Die affizierende Wirkung von Patient $O$ erklärt sich z. B. auch daraus, dass einst die aus der irischen Unterschicht stammende ,Typhoid Mary', obgleich selbst frei von Symptomen, immer wieder Mitglieder wohlhabender Familien mit Typhus infiziert haben soll und dafür öffentlich gescholten und mit lebenslanger Quarantäne belegt wurde. Die CoronaKinder rühren nicht nur aufgrund eines biologisch wirksamen Kindchenschemas, sondern auch, weil die Figur der, verlorenen Generation' seit dem Ende des Ersten Weltkriegs regelmäßig für Gesellschaftsdiagnosen bemüht wird und eine dunkle Zukunft vorhersagbar zu machen glaubt.

Kurzum: Auch wenn wir im Folgenden den Figuren den Vortritt lassen, so schauen wir auf sie mit einem Interesse für die gesellschaftlichen Erfahrungen und Fragen, die sie artikulieren, aber auch für die Lösungen, die sie in ihren Geschichten anbieten. Die Runde der Autor*innen setzt sich aus Kulturwissenschaftler*innen verschiedener disziplinärer Herkunft zusammen. Unsere Blog-Reihe verknüpft diese verschiedenen fachlichen Expertisen und eröffnet mit ihren sozialfigurativen Skizzen auch hinsichtlich der Schreibstile und Beschreibungsformen einen Experimentierraum, der zu Kommentaren anregen soll.

\section{References}

1. https://www.berliner-zeitung.de/politik-gesellschaft/verluste-muessenartikuliert-werden-duerfen-li.86049 
2. Vgl. Moebius, Stefan/ Markus Schroer (Hrsg.) (2010): Diven, Hacker, Spekulanten. Sozialfiguren der Gegenwart. Suhrkamp; Moser, Sebastian J./Schlechtriemen, Tobias (2018): „Sozialfiguren - zwischen gesellschaftlicher Erfahrung und soziologischer Diagnose“, Zeitschrift für Soziologie, No. 3: 164180. https://doi.org/10.1515/zfsoz-2018-1011

3. Elias, Norbert (1983): „Über den Rückzug der Soziologen auf die Gegenwart“, Kölner Zeitschrift für Soziologie und Sozialpsychologie , 35: 29-40.

SUGGESTED CITATION: Moser, Sebastian J.; Schlechtriemen, Tobias: Sozialfiguren der Corona-Pandemie. Ein Aufschlag, in: KWI-BLOG, [https://blog.kulturwissenschaften.de/sozialfiguren-der-corona-pandemie/], 16.11.2020

DOI: https://doi.org/10.37189/kwi-blog/20201116-0900 


\section{DuEPublico}

Dieser Text wird über DuEPublico, dem Dokumenten- und Publikationsserver der Universität Duisburg-Essen, zur Verfügung gestellt. Die hier veröffentlichte Version der EPublikation kann von einer eventuell ebenfalls veröffentlichten Verlagsversion abweichen.

DOI: $\quad$ 10.37189/kwi-blog/20201116-0900

URN: urn:nbn:de:hbz:464-20201116-091944-8 